\title{
Emittance growth and energy loss due to coherent synchrotron radiation in a bunch compressor
}

\author{
H. H. Braun, R. Corsini, L. Groening, and F. Zhou \\ European Organization for Nuclear Research, CH-1211 Geneva 23, Switzerland \\ A. Kabel and T. O. Raubenheimer \\ Stanford Linear Accelerator Center, Menlo Park, California 94025 \\ R. Li \\ Thomas Jefferson National Accelerator Facility, Newport News, Virginia 23606 \\ T. Limberg \\ Deutsches Elektronen Synchrotron, D-22607 Hamburg, Germany \\ (Received 25 September 2000; published 13 December 2000)
}

\begin{abstract}
Electron bunches of high charge (up to $10 \mathrm{nC}$ ) are compressed in length in the Compact Linear Collider Test Facility magnetic chicane to less than $0.4 \mathrm{~mm}$ rms. The short bunches radiate coherently in the chicane magnetic field, and the horizontal and longitudinal phase space density distributions are affected. This paper reports the results of beam emittance and momentum measurements. Horizontal and vertical emittances and momentum spectra were measured for different bunch compression factors and bunch charges. In particular, for $10 \mathrm{nC}$ bunches, the mean beam momentum decreased by about 5\% while the rms momentum spread increased from $2 \%$ to $8 \%$. The experimental results are compared with simulations made with the code TRAFIC4.
\end{abstract}

PACS numbers: 29.27.Bd, 41.60.Ap, 41.75.Lx

\section{INTRODUCTION}

Short electron bunches traversing a dipole with bending radius $\rho$ can emit coherent synchrotron radiation (CSR) at wavelengths longer than the bunch length. The enhancement of the radiated power, with respect to the classical synchrotron radiation, can be expressed as [1]

$$
\Delta P_{\mathrm{coh}} \approx 0.028 N^{2} \frac{c e^{2}}{\epsilon_{0} \rho^{2 / 3} \sigma_{l}^{4 / 3}},
$$

where a longitudinal Gaussian distribution of the $N$ particles with constant rms bunch length $\sigma_{l}$ along the curved trajectory is assumed. The CSR induces an average momentum loss and a momentum spread on the bunch. For relativistic beams the momentum loss is independent of the beam energy and the effect can be treated like a wakefield. Since it takes place in a dispersive region, the transverse phase space distribution is also affected, and the beam emittance in the bending plane increases. These effects are a concern in all accelerator applications in which high-charge short bunches are needed, e.g., free electron lasers, linear colliders, and two-beam accelerators. Analytical treatments have been developed for idealized conditions [1-4], but for many practical cases the effect must be treated numerically. Some codes have been developed for this purpose [5,6], but a complete benchmark of the codes with measurements is lacking thus far. The parameters of the drive beam in the second Compact Linear Collider Test Facility (CTF II) [7] are well suited to study the CSR effects experimentally.

CTF II (see Fig. 1) was built to demonstrate the feasibility of two-beam acceleration at $30 \mathrm{GHz}$. The high-charge drive beam is generated in a $3 \mathrm{GHz}$ rf gun, and accelerated in two traveling wave structures (TWS). The drive beam bunches are then compressed in a magnetic chicane and generate $30 \mathrm{GHz}$ rf power in a series of power extraction

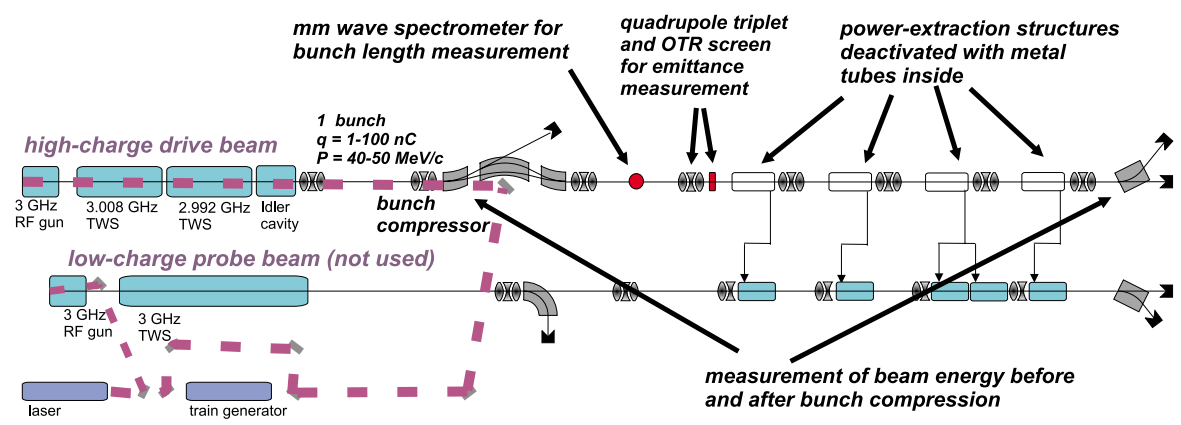

FIG. 1. (Color) The CTF II. The CSR experiments were performed using the drive beam line (upper part). 
and transfer structures (PETS). This power is used to accelerate a low-charge probe beam in a parallel beam line.

Only the drive beam line of CTF II has been used to perform the CSR experiment. Single bunches with charges of 5 and $10 \mathrm{nC}$ have been used. For both charges, the horizontal and vertical beam emittances and the momentum spectra of the drive beam have been measured as a function of the deflection angle in the chicane. Similar measurements have been performed in CTF II in the past, but without measurements of beam spectra [8]. The results of simulations made using the code TRAFIC4[5] are reported for comparison.

\section{EXPERIMENTAL SETUP}

Bunch compression is used in CTF II to enhance the $30 \mathrm{GHz}$ power production, and is achieved by acceleration off crest (such that particles at the tail of the bunch have higher energies than those at the head), in combination with a magnetic chicane composed of three rectangular dipoles as shown in Fig. 2. The deflection angle in the first and the last dipole can be varied from $3.7^{\circ}$ to $14^{\circ}$ (twice these values in the central dipole). The corresponding momentum dependence of the path length $R_{56}=\delta s /(\delta p / p)$ ranges from 6 to $90 \mathrm{~mm}$. Because of the construction of the vacuum chamber, the chicane cannot be switched off completely. Using this scheme, rms bunch lengths of less than $0.4 \mathrm{~mm}$ can by achieved for bunch charges of $10 \mathrm{nC}$.

The beam momentum spectra were measured at the entrance to the chicane by switching off the last two chicane magnets and using the first one as a spectrometer. A second spectrometer at the end of the line permits the measurement of the beam spectra after the passage in the chicane. The PETS were deactivated by shielding them with metallic tubes, in order not to perturb the momentum distribution. Two wall-current monitors placed in front of the chicane and in front of the second spectrometer measured the beam intensities, in order to monitor the losses.

The bunch lengths were measured behind the chicane by analyzing the frequency spectrum of the mm-wave

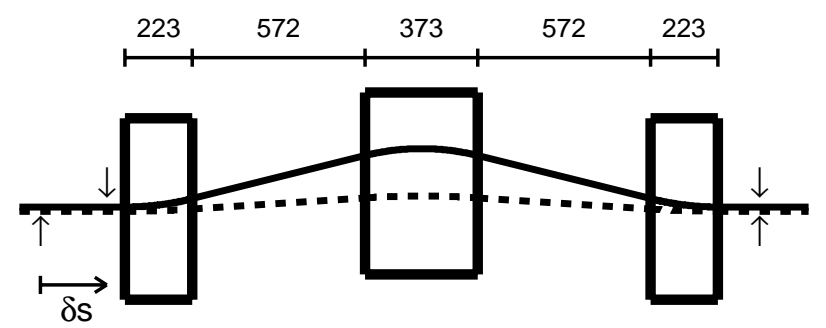

FIG. 2. The bunch compressor chicane of CTF II consists of three rectangular dipole magnets. The solid and dotted lines show the trajectory of a particle with mean momentum $p_{0}$ and a momentum of $p_{0}+\delta p$, respectively. It introduces a path length difference $-\delta s$ to particles with a momentum deviation of $\delta p$ with respect to the mean momentum $p_{0}$. The effective magnetic lengths in $\mathrm{mm}$ are given as well. radiation excited by the beam passing an rf waveguide connected to the vacuum chamber [9]. Transverse beam profiles were recorded after the chicane using an optical transition radiation (OTR) screen and a camera. The horizontal and vertical emittances were measured simultaneously by using the quadrupole scanning technique. In order to cover the full range of phase advances from $0^{\circ}$ to $180^{\circ}$ in both transverse planes, the three quadrupoles of a triplet upstream of the OTR screen were varied independently. For accurate determination of the beam widths, only those profiles which fit well on the OTR screen were selected. The off-crest rf phase and the range of chicane settings were selected in order to achieve overcompression of the initial bunch length, thus covering a sufficient range in bunch lengths.

\section{MOMENTUM SPECTRA AND BUNCH LENGTHS}

The beam momentum spectra measured for different bunch compressor settings are shown in Fig. 3 ( $5 \mathrm{nC}$ case) and Fig. 4 (10 nC case). The spectra from TRAFIC4 simulations are also shown. Since the spectrometer at the end of the beam line consists of a deflecting dipole it also represents a source of CSR, and the spectra result from the sum of the CSR effects in the compressor chicane and in the spectrometer. As the bunches are compressed, the spectrum broadens, and in the $10 \mathrm{nC}$ case it develops two maxima beyond maximum compression. During overcompression the spectrum narrows again.

The measured bunch lengths and the mean momenta and rms momentum spreads calculated from the measured spectra are plotted in Figs. 5 and 6 as functions of $R_{56}$. They are compared with the results of the TRAFIC4 simulations. For $5 \mathrm{nC}$, no significant changes of the mean

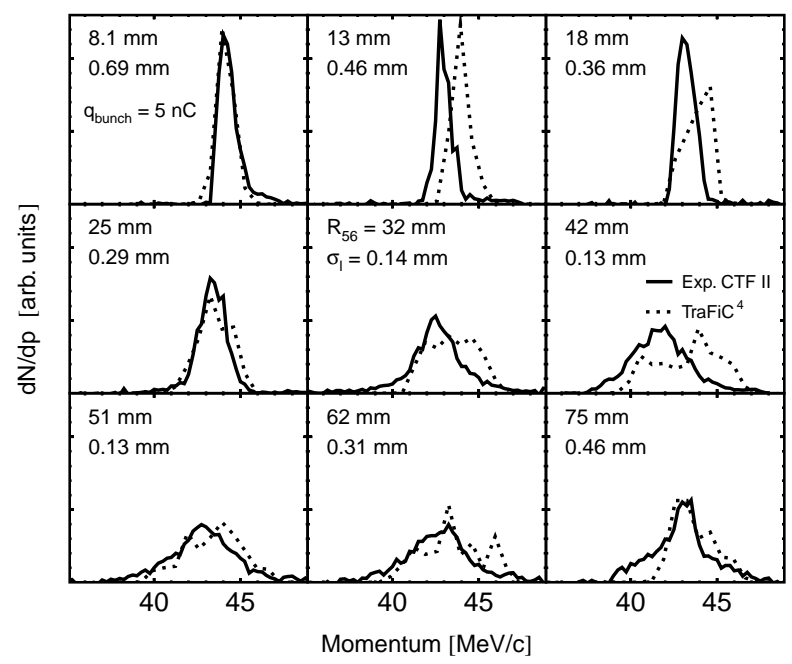

FIG. 3. Measured (solid line) and calculated (dotted line) momentum spectra at the end of the beam line for different compressor settings $R_{56}$ (upper values), with a bunch charge of $5 \mathrm{nC}$. The measured rms bunch lengths are also shown (lower values). 


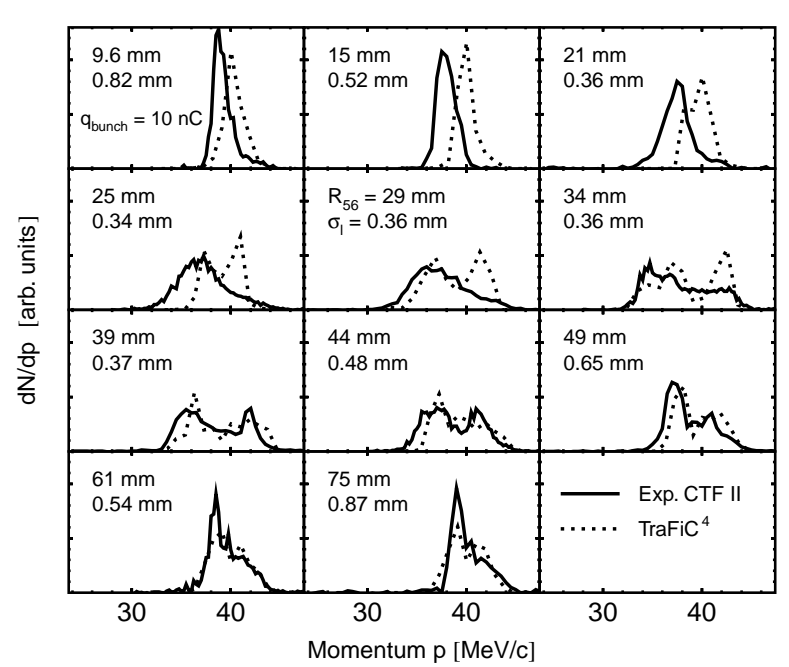

FIG. 4. Measured (solid line) and calculated (dotted line) momentum spectra at the end of the beam line for different compressor settings $R_{56}$ (upper values), with a bunch charge of $10 \mathrm{nC}$. The measured rms bunch lengths are also shown (lower values).

beam momentum were observed, although the momentum spread increased by a factor of 4 at full compression with respect to the initial spread, and decreased to a factor of 3 larger than the initial spread at overcompression. For charges of $10 \mathrm{nC}$, a decrease of the mean momentum by $2 \mathrm{MeV} / c$, i.e., $5 \%$ of the initial beam momentum, was measured at full compression. The momentum spread shows a behavior similar to the one described for $5 \mathrm{nC}$. In the $5 \mathrm{nC}$ case, the dependence of the measured bunch length on $R_{56}$ shows a symmetric shape as expected from linear longitudinal dynamics. On the other hand, the asymmetric shape of the corresponding curve for $10 \mathrm{nC}$ indicates a strong impact of CSR on the longitudinal properties for $R_{56}>20 \mathrm{~mm}$.

Using the program TRAFIC4, the bunch, represented by 500 macroparticles, was tracked from the entrance to the chicane to the end of line spectrometer dipole. Each of the two dimensional macroparticles had a longitudinal and a vertical extension. Their electromagnetic fields were calculated from first principles by applying the Maxwell equations. In order to obtain self-consistent simulations, the code starts with two identical bunches. The macroparticles comprising the first bunch are tracked on a small increment of the beam line by applying the external magnetic fields only. In the next step, the macroparticles of the second bunch are tracked along the same beam line increment by applying the external fields and the fields generated by the first bunch. For the following beam line increments, a leapfrog algorithm is applied, which tracks one bunch according to the sum of the external fields and the fields generated by the bunch tracked in the previous step. If the beam line increments are chosen to be infinitely small, the trajectories of the two bunches are identical and the simulation is fully self-consistent. In practice, a com-

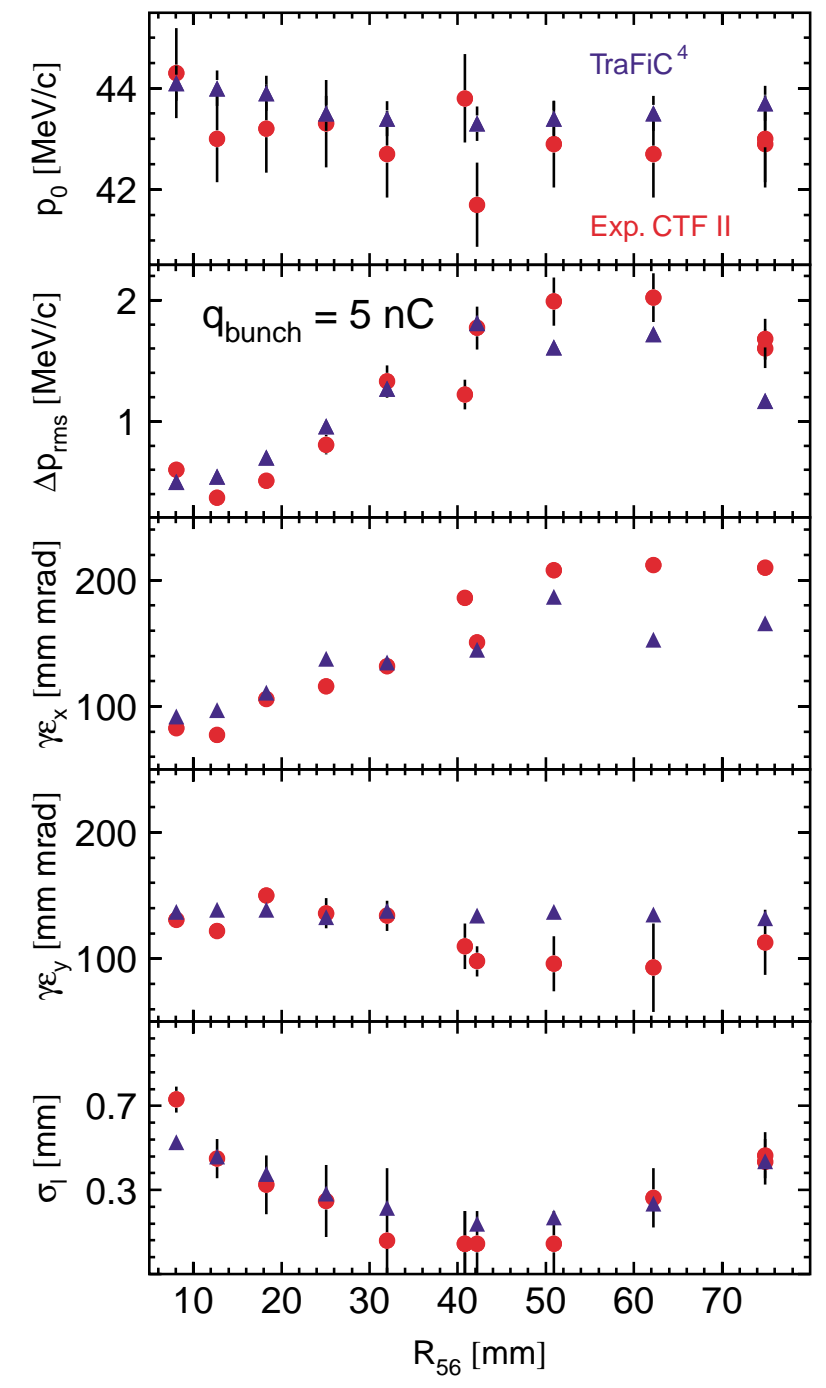

FIG. 5. (Color) Measured (circles) and calculated (triangles) rms bunch lengths, normalized transverse emittances, momentum spreads, and mean momenta as functions of $R_{56}$ for a bunch charge of $5 \mathrm{nC}$.

promise between accuracy and calculation time must be found. After tracking, the resulting bunch lengths, emittances, and momentum spectra were calculated from the final macroparticle distributions and are shown in Figs. 5 and 6 as well.

In order to determine the initial conditions to be used in the simulations, the bunch lengths measured after the chicane have been used together with the momentum spectra recorded at the entrance to the chicane. The initial phase space distributions in the longitudinal plane have been reconstructed taking into account the contribution to the intrabunch momentum correlation of the off-crest $\mathrm{rf}$ phase and of the short-range longitudinal wakefields in the accelerating structures. The initial bunch length has been chosen to fit the momentum spectra at the chicane entrance and the measured bunch lengths, assuming that CSR does not influence the bunch length. As mentioned 


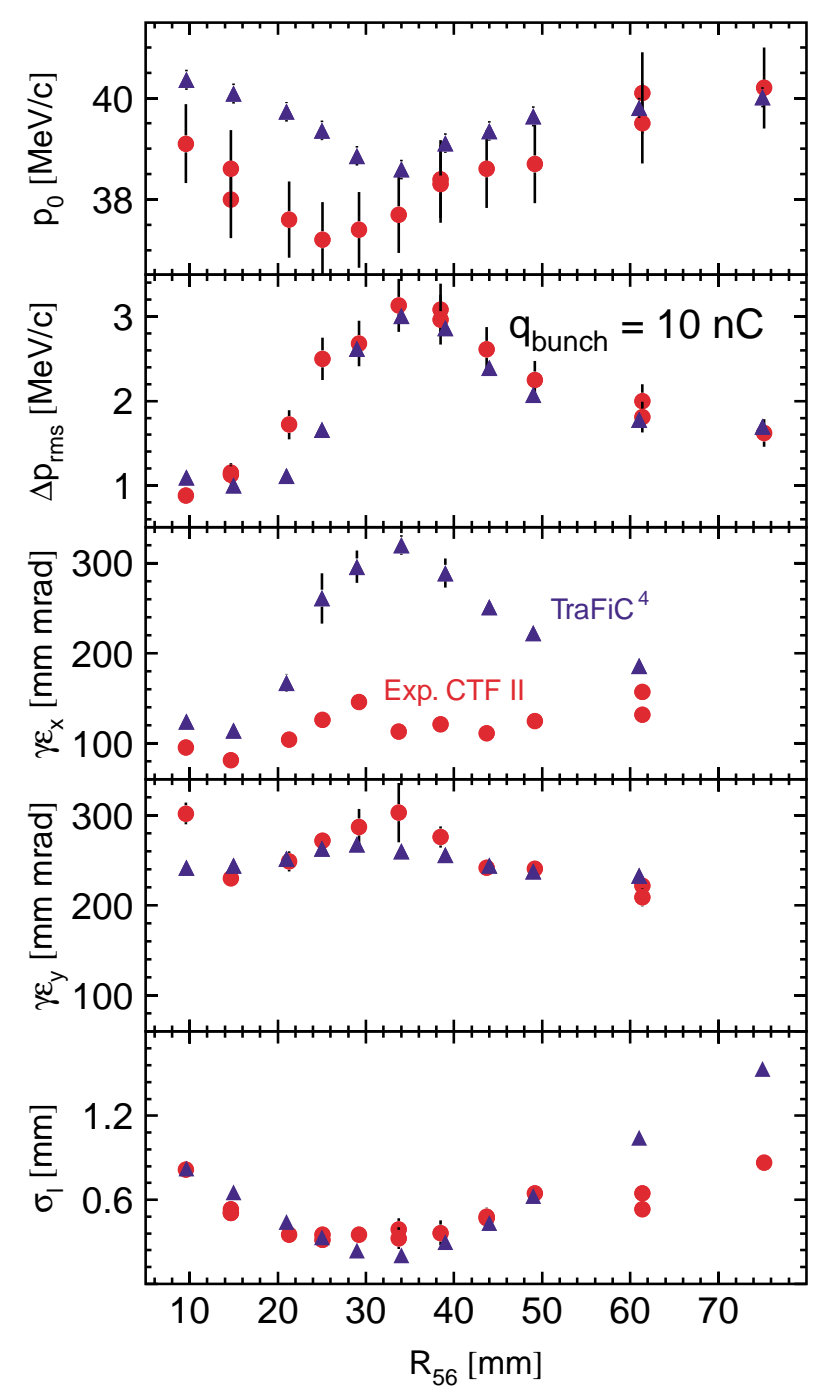

FIG. 6. (Color) Measured (circles) and calculated (triangles) rms bunch lengths, normalized transverse emittances, momentum spreads, and mean momenta as functions of $R_{56}$ for a bunch charge of $10 \mathrm{nC}$.

above, this assumption seems to be justified for only $R_{56}<$ $20 \mathrm{~mm}$ in the $10 \mathrm{nC}$ case. Indeed, while a linear longitudinal model describes well the measured bunch length dependence from $R_{56}$ for $5 \mathrm{nC}$, no set of initial conditions could be found that can describe the measured values for $10 \mathrm{nC}$. Therefore, only the bunch lengths for small $R_{56}$ have been used in this case. Since only a few measurement points are available for the fit, the input parameters for the $10 \mathrm{nC}$ measurements are known with less accuracy.

In the case of $5 \mathrm{nC}$, the dependence of the observables on $R_{56}$ was well reproduced. For $10 \mathrm{nC}$, the calculated bunch lengths agreed with the measured ones until full compression, but the asymmetric shape of the bunch length curve was not reproduced, although the TRAFIC4 simulation looks more accurate than the simple linear calculation. The amount of momentum loss for $10 \mathrm{nC}$ was calculated correctly, but the experimental and theoretical curves are shifted with respect to each other, possibly indicating different initial longitudinal beam parameters in the experiments and the simulations. Another possible explanation is a fluctuation in the initial beam parameters while the measurement was taken. In particular, variations in rf power and phase could be invoked, since the same initial conditions have been used to simulate all of the measurement points and a phase variation of a few degrees would be enough to explain the discrepancies found.

On the other hand, a very good matching of the measured and calculated momentum spreads was found for both cases. In the case of $5 \mathrm{nC}$, the simulations reveal no splitting of the maximum and the shapes of the spectra show a good agreement. In the $10 \mathrm{nC}$ case, although the rms widths are in good agreement, the measured and simulated momentum spectra have somewhat different shapes. The formation of two maxima occurs in the simulations for smaller values of $R_{56}$. Since the CSR wakefield depends on the exact longitudinal charge distribution within the bunch, the details of the momentum spectrum shape should depend on that as well.

In the simulation, an initial Gaussian charge distribution has been assumed, but previous bunch length measurements with a streak camera indicate a different, asymmetric charge distribution. The simulations show that the splitting of the maxima in the spectra for $10 \mathrm{nC}$ is due to CSR in the chicane, since such a feature is present in the simulated spectrum right after the chicane.

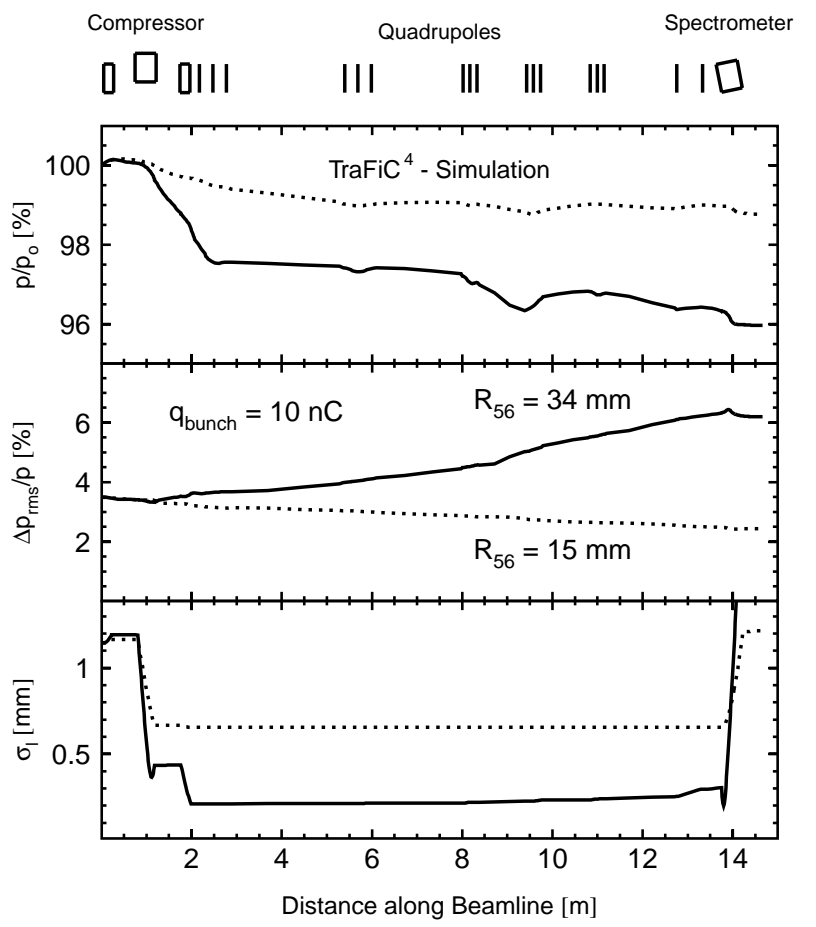

FIG. 7. Evolution of the bunch length, the momentum spread, and the mean momentum along the CTF II beam line as simulated with TRAFIC4 for $10 \mathrm{nC}$. The results with two different settings of the chicane are shown, corresponding to $R_{56}=34 \mathrm{~mm}$ (solid line) and $R_{56}=15 \mathrm{~mm}$ (dotted line). 
On the other hand, the broadening of the spectra seems to be caused by space charge during the drift to the spectrometer. This is apparent in Fig. 7, where the evolution of the bunch length, the momentum spread, and the momentum loss along the beam line, simulated with TRAFIC4, are shown.

In spite of the fact that some features of the simulated momentum loss curve are not yet understood, it can be seen from Fig. 7 that the momentum loss is mainly concentrated in the chicane region where the bunches are short, and is a direct indication of CSR emission. On the other hand, for $R_{56}=34 \mathrm{~mm}$, the momentum spread increases along the whole beam line after the chicane, showing that such growth is not directly caused by a CSR effect.

\section{TRANSVERSE EMITTANCES}

The measured horizontal and vertical emittances after the chicane are plotted in Fig. 5 for $5 \mathrm{nC}$ and in Fig. 6 for $10 \mathrm{nC}$ as functions of $R_{56}$, together with the bunch lengths. The measured vertical emittances for $5 \mathrm{nC}$ are constant within the error bars, and are consistent with the simulation results. The measured horizontal emittances for $5 \mathrm{nC}$ increase until full compression, then a saturation occurs. The four highest values seem to be shifted with respect to the lower values. This shift might be due to dispersion at the location of the OTR screen, caused by a mismatch of the relative magnetic strengths of the chicane dipoles. It must be noted that these four points were recorded after switching off the last two magnets of the chicane in order to check the beam momentum, and then switching on the magnets again.

For $10 \mathrm{nC}$, the measured vertical emittance shows a maximum at full compression. This qualitative behavior is also found in the simulations. The measured data points for the horizontal emittance scatter after overcompression, thus not allowing meaningful conclusions. The huge growth of the horizontal emittance predicted by the simulations was not found experimentally. It can be noted that the increase of the momentum spread also affects the accuracy of the emittance measurements and contributes to the error bars and the scattering of the data points.

\section{CONCLUSIONS AND OUTLOOK}

The measurements made in the CTF II drive beam line showed clear signs of CSR emission. In particular, the momentum loss and bunch length as a function of the bunch compressor setting in the $10 \mathrm{nC}$ case are well explained by the CSR effect, such as the two-peak momentum spectrum shape at $10 \mathrm{nC}$. A good agreement was found between measurements and simulations made with the TRAFIC4 code for all observables, except the emittance growth for bunches of $10 \mathrm{nC}$, which is not yet understood.

After investigating the dependence of the CSR effects on the bunch length, a series of experiments on the dependence on the transverse optics in the compressor chicane is under way at CTF II. The triplet before and after the chicane will be used to obtain a transversely uncorrelated beam (i.e., $\alpha_{x, y} \equiv 0$ ) in the chicane center, respectively. Outside the chicane region, the optics will not be changed for all settings. The emittance growth will be measured as a function of the horizontal beam size while leaving the vertical beam dimensions constant. Future experiments will aim, in particular, at an experimental investigation of the shielding effect of the beam pipe on the CSR emission. The extension of the CSR spectrum is limited at the high-frequency end by the bunch length, and at the lowfrequency end by the cutoff of the beam pipe. If the beam pipe dimensions are small with respect to the bunch length, CSR emission is suppressed. While this is indeed the case in many accelerators, in the CTF II chicane the free space approximation is valid. It is interesting to explore the intermediate regime since often only a partial shielding can be used to reduce the unwanted CSR effect in the case of very small bunches. Several approaches to the calculation of the shielding effect exist [1-4,10], using different approximations. The TRAFIC4 code is also capable of treating the shielding, and could be benchmarked against the measurements.

Short bunches will be passed through an additional fourmagnet chicane installed downstream of the bunch compressor. Three vacuum chambers of different heights will be used in order to provide different shielding environments. The new chicane has been designed for large deflection angles and small dispersion functions, i.e., small $R_{56}$. Therefore, the bunch length can be made short and relatively constant in the new chicane, while CSR emission in this region will be high. In order to allow for an accurate measurement of the initial beam parameters it can be switched off completely. A further advantage will be the smaller disturbance from possible dispersion errors.

[1] J. B. Murphy, S. Krinsky, and R. L. Gluckstern, Part. Accel. 57, 9 (1997).

[2] L. I. Schiff, Rev. Sci. Instrum. 17, 6 (1946).

[3] J. S. Nodvick and D. S. Saxon, Phys. Rev. 96, 180 (1954).

[4] S. A. Kheifets and B. Zotter, CERN Report No. CERN-SL 95-43 (AP), 1995.

[5] A. Kabel, M. Dohlus, and T. Limberg (to be published).

[6] R. Li, in Proceedings of the 6th European Particle Accelerator Conference, Stockholm, 1998 (Institute of Physics, Bristol, UK, 1998).

[7] H. H. Braun, in Proceedings of the 7th European Particle Accelerator Conference, Vienna, 2000, http://accelconf. web.cern.ch/AccelConf/e00/index.html

[8] H. Braun, F. Chautard, R. Corsini, T. O. Raubenheimer, and P. Tenenbaum, Phys. Rev. Lett. 84, 658 (2000).

[9] H. H. Braun and C. Martinez, in Proceedings of the 6th European Particle Accelerator Conference, Stockholm, 1998 (Ref. [6]).

[10] R. L. Warnock and K. Bane, SLAC Report No. SLACPUB-95-6837, 1995. 